\title{
El aprendizaje cooperativo, una aproximación a la tutoría entre iguales en la formación de los estudiantes de la Licenciatura en Educación Preescolar y Física del BINE
}

The cooperative learning, an approach to peer tutoring in the training of students in the Bachelor of Early Childhood Education and BINE

César Simoni Rosas

Benemérito Instituto Normal del Estado Puebla caef.lef.bine2010@gmail.com

Hadi Santillana Romero Benemérito Instituto Normal del Estado Puebla caef.lef.bine2010@gmail.com

Alejandra Díaz Ramírez Benemérito Instituto Normal del Estado Puebla aledira.ram@gmail.com

\section{Resumen}

En la actualidad, un sin fin de evaluaciones arrojan resultados poco satisfactorios en la educación básica, lo que nos mira a centrar nuestra mirada en los docentes; es así como la educación normal juega un papel esencial en la formación de una sociedad que responda a las necesidades de un mundo en constante cambio. La educación normal ha sufrido una evolución para ser considerada parte del nivel superior, sin embargo, al analizar su esencia y relevancia es imperativo reconocer que deben existir cambios medulares en las prácticas educativas de los docentes de las normales que doten a los docentes en formación de saberes basados en nuevas tendencias que no se limiten a un discurso vanguardista sino 
que reflexionen a partir de su trabajo cotidiano para educar a las generaciones futuras en ambientes cooperativos competentes.

Palabras Clave: Formación docente, tutoría entre iguales, aprendizaje colaborativo, prácticas educativas.

\section{Abstract}

Today, countless evaluations yield unsatisfactory results in basic education, which we look to focus our attention on teachers, this is how normal education plays an essential role in the formation of a society that meets the needs of a changing world. The regular education has undergone an evolution to be considered part of the upper level, however, to analyze its essence and relevance is imperative to recognize that there must be deep changes in the educational practices of teachers in normal equip teachers in training knowledge based on new trends that are not limited to a discourse reflect edgy but from their daily work to educate future generations competent cooperative environments.

Key words: Teacher training, peer tutoring, collaborative learning, educational practices.

Fecha recepción: Enero 2011 Fecha aceptación: Mayo 2011

\section{Introducción}

La reforma educativa actual a nivel mundial enfatiza los procedimientos, valores y actitudes que constituyen el currículo y enmarcan la intervención educativa; en la enseñanza normalista ésta postura reviste un compromiso mayor al reconocer que el currículo y actuar docente integran la base sobre la cual los futuros educadores desarrollan sus competencias. Por otro lado, la calidad educativa está estrechamente vinculada con la capacidad que una institución educativa tiene para dar respuesta a la 
diversidad de alumnos y alumnas que atiende, esto exige una intervención docente diversificada y globalizadora que atienda a necesidades diferentes. En esta línea la relevancia del aprendizaje en grupo es muy alta, el trabajo en grupo, la formación de equipos de aprendizaje, el desarrollo de actitudes de cooperación, constituyen una variable sustancial de formación básica en la Enseñanza Superior, de cara a una mejora en la calidad educativa de la enseñanza preescolar y de la educación física.

Bajo estas premisas las metodologías activas de enseñanza se vislumbran como eje central del proceso formativo del alumnado, en específico la aplicación de los principios que sustentan el aprendizaje cooperativo se plantean en la presente propuesta como una opción para fortalecer la formación de los estudiantes normalistas de las licenciaturas en educación física y preescolar está enmarcada en las asignaturas de Observación y Práctica Docente III y IV así como del Seminario de Análisis del Trabajo Docente I y II, debido a que ambos cursos se consideran medulares en la línea de acercamiento a la práctica porque en ellas se integran no sólo los saberes didácticos y metodológicos que debe poseer un docente sino también se analiza la manera en que la intervención docente debe estar fundamentada por un estudio profundo del desarrollo del niño y el adolecente para proponer practicas docentes que atiendan las necesidades e intereses de los educandos. A partir de ello se planteó el objetivo de analizar las características de las estrategias basadas en el trabajo cooperativo, a fin de promover mejorar la intervención docente de los estudiantes normalista de las licenciaturas antes mencionadas y con ello fortalecer los aprendizajes significativos en el proceso de enseñanza-aprendizaje de los niños y adolescentes de preescolar y educación física atendidos en las escuelas de práctica.

A partir de 1999 la educación normal ha establecido en los lineamientos del Plan de estudios la construcción un perfil de docente basado en el desarrollo de habilidades intelectuales específicas, competencias docentes, el dominio de los contenidos de enseñanza y propósitos de la educación básica que les permita responder con una alta identidad ética y profesional a las condiciones sociales del entorno de la escuela; sin 
embargo y tras una década de la puesta en práctica de ésta reforma se denota que dicha consolidación no ha correspondido a los estilos y prácticas escolares que en la actualidad deben promoverse. Retomando las palabras de Carles Monereo (2009), "Somos profesores del siglo XX, enseñando a estudiantes del siglo XXI con un metodología del siglo XIX" entendemos el gran reto que enfrentan las normales al buscar formar futuros docentes que enseñarán a las generaciones del mañana con experiencias y competencias emanadas de un siglo con características diferentes a las que los alumnos enfrentarán, de allí que la docencia requiere una transformación que sobre todo implique una nueva identidad de la tarea docente que atienda las diferentes necesidades de los estudiantes propiciando relaciones grupales en las cuales las respuestas adecuadas no partan sólo del educador sino que se facilitan desde los mismos alumnos.

Indiscutiblemente, los rasgos del perfil docente son el referente principal para que las comunidades educativas normalistas dispongan de criterios para valorar el avance del plan y los programas, la eficacia del proceso de enseñanza y de los materiales de estudio, el desempeño de los estudiantes, así como las demás actividades y prácticas realizadas en cada institución. Para cumplir con los propósitos formativos planteados, el mapa curricular abarca áreas de actividades de formación, entre las que destacan las actividades de acercamiento a la práctica escolar que en el plan de estudios se desarrollan en los primeros seis semestres combinando el trabajo directo en los jardines de niños con la preparación de las estancias y el análisis de las experiencias obtenidas, que se realizan en la escuela normal. Del mismo modo y para dar continuidad a este proceso formativo, la práctica intensiva en condiciones reales de trabajo se realiza en el séptimo y octavo semestres, en ella, los estudiantes se hacen cargo de un grupo de educación preescolar o física con la asesoría continua de un maestro tutor, además de contar con el apoyo y seguimiento del personal docente de la escuela normal.

Revisando los resultados de la evaluación realizada por el Centro Nacional de Evaluación para la Educación Superior (CENEVAL) en más de cinco generaciones a la aplicación de los 
planes y programas de estudio; en el 2010 se han reiterado los resultados poco satisfactorios entorno a las competencias didácticas que oscilaron entre un $44.58 \%$ para el octavo semestre de la licenciatura en educación física y el 59.05\% del octavo semestre de la licenciatura en educación preescolar; estos saberes se caracterizan por que los futuros docentes reconozcan las diferencias individuales de los educandos que intervienen en los procesos de aprendizaje y aplica estrategias didácticas para estimularlos; así como la capacidad de establecer un clima de relación en el grupo, que favorezca actitudes de confianza, autoestima, respeto, orden, creatividad, curiosidad y placer por el estudio, así como el fortalecimiento de la autonomía de los educandos. En el mismo sentido, la identidad profesional y ética a partir de la cual los docentes promueven el valor del trabajo en equipo como un medio para la formación continua y el mejoramiento de la escuela, a partir de poner en juego actitudes favorables para la cooperación y el diálogo con sus colegas en el caso de educación física encontramos un $46.89 \%$ para el sexto semestre y $42.43 \%$ para el octavo (tabla 1 ).

La problemática descrita anteriormente se agudiza al referirnos a los hallazgos generados a partir de las visitas de seguimiento a los estudiantes de ambas licenciaturas entre las que destacan:

1. Intervenciones didácticas que propician el individualismo entre los niños y adolescentes.

2. Prácticas educativas centradas en el docente que restringen la ayuda mutua, el dialogo entre los niños, las metas comunes y la autonomía del educando.

3. Desconocimiento de los principios del aprendizaje estratégico que se percibe a través de una disonancia entre lo planeado y lo llevado a la práctica.

En esencia encontramos estudiantes con un discurso basado en los preceptos de Vygotsky y el aprendizaje social con una didáctica tradicional producto de la enseñanza bajo la cual han sido formados, tal como lo refiriera Monereo (2008) el modelado que realizamos los 
docentes en la escuela normal y los profesores de educación básica que están a cargo de los grupos de práctica ejercen una influencia mayor que el análisis de diversos artículos a lo largo de los estudios de formación inicial. En este proceso de convergencia la actividad docente exige adquirir nuevos enfoques, lo que presume implicaciones directas en la metodología docente (ZABALZA, 2000; MAYOR, 2003), así es como consideramos fundamental el conocimiento y utilización de otras técnicas o estratégicas metodológicas que aseguren el proceso de enseñanza-aprendizaje, en el que el alumno sea el eje fundamental, en torno al cual gire el diseño de situaciones didácticas que propicien las interacciones educativas.

De esta manera y en palabras de Herrera que refiere a Díaz (1998), los componentes intencionales, conceptuales y comunicativos que ocurren durante las interacciones docente - alumno y alumno - alumno, se convierten en los elementos básicos que permiten entender los procesos de construcción de un conocimiento que es compartido. La capacidad para aprender de forma cooperativa, de trabajar en equipo, de buscar el consenso, de enriquecerse a partir de las aportaciones del otro; la capacidad de comunicación verbal, la responsabilidad, y otros muchos saberes son competencias de ninguna manera escapan al quehacer docente por lo que la intención de buscar un profesor reflexivo (BLÁNDEZ, 1995) y (1996), con el cambio de orientación que supone la acción reflexiva (IMBERNÓN, 2002); que a su vez permita asumir la necesidad de desarrollar la capacidad crítica para que sea ésta el motor de cambio social al que se desea llegar desde la formación del profesorado, en palabras de Ovejero (1990); asumir lo significativo que resulta aprender a partir de la necesidad de enseñar a los demás, son la base del aprendizaje cooperativo.

El aprendizaje cooperativo proyecta un modo alternativo de organizar los procesos cognitivos en el proceso de enseñanza aprendizaje tanto dentro como fuera del aula, con su implementación se busca superar la aplicación de técnicas tradicionales de aprendizaje grupal, interesadas más por los resultados que por rendimientos, responsabilidades 
grupales más que individuales, grupos homogéneos más que heterogéneos, líderes únicos en vez de liderazgos compartido. En definitiva, todos estos argumentos sumados a la convicción de que el futuro profesor debe caracterizarse por el alto grado de compromiso con los demás, que asuma a través de un proyecto compartido la necesidad de romper el aislamiento en el que muchos docentes viven su actividad profesional.

Es por ello que los métodos y técnicas de aprendizaje cooperativo, presuponen cinco elementos esenciales: interdependencia positiva, interacción cara a cara, responsabilidad individual, habilidades sociales y el procesamiento grupal autónomo (JOHNSON y JONHSON 1985, 1989). Existen múltiples ventajas del uso de las técnicas de aprendizaje cooperativo en educación, contrastadas en numerosos trabajos de investigación anteriores y a partir de la revisión de las mismas se empleó la tutoría entre iguales y la enseñanza reciproca a través de rompecabezas o Puzzle (ARONSON, 1978) y carrusel.

En la técnica del Puzzle, el profesor divide o fragmenta el contenido a abordar en tantas secciones o estaciones como miembros compongan el grupo de trabajo, cada alumno se encargará estudiar y conocer su parte, posteriormente se reúnen en "grupos de expertos" con alumnos que han realizado la misma tarea, para compartir la información; por último, los alumnos vuelven a sus grupos de origen para integrar conjuntamente todo el material.

Recuperando los lineamientos de la investigación- acción se analiza la propuesta que inició en el 2010-2011 con veintiún estudiantes de séptimo y octavo semestres de la licenciatura en educación preescolar y física con quienes se inició la tutoría entre iguales en un taller de lectura y en las sesiones de seminario de análisis del trabajo docente. Los estudiantes fungieron como tutores de sus iguales en la revisión de los diarios de trabajo a través del cual se destacó el trabajo en equipo de carácter interdisciplinar, la adaptación a nuevas situaciones, el compromiso ético y la capacidad de análisis y síntesis, así como el desarrollo de habilidades de comunicación oral y escrita a través de la TIC, debido a que 
los estudiantes tenían diferentes periodos de trabajo docente, lo que impedía que las sesiones grupales se desarrollaran físicamente de manera constante.

A la par, los estudiantes recuperaron los principios del trabajo cooperativo en su intervención en los jardines de niños y primarias, producto de estas experiencias los estudiantes generaron sus documentos recepcionales al aplicar el puzzle a los circuitos de acción motriz en las sesiones de educación física en la escuela primaria y la enseñanza reciproca en el fomento de la lengua escrita y el pensamiento reflexivo en los niños preescolares.

La primer etapa de esta propuesta ha sentado un precedente para establecer una segunda fase en la que diez estudiantes del séptimo semestre de la licenciatura en educación preescolar son tutoras de 24 estudiantes de quinto semestre apoyando el proceso de diseño de actividades didácticas y elaboración de diario de trabajo, ésta actividad ha permitido garantizar que cada alumna de quinto año reciba un acompañamiento en el proceso de planeación docente, mientras que las estudiantes de séptimo semestre han asumido su rol requiriendo fortalecer algunos contenidos educativos que conocían, sin embargo, al tener que explicarlos han tenido que releer, estudiar, discutir y reflexionas sobre sus saberes a fin de desempeñar cada vez más su rol de tutor. Una acción importante en el proceso versa en que las estudiantes están diseñando actividades con una estructura cooperativa que ha permeado en el diseño de las actividades didácticas tal como se refiere en la planeación de la estudiante Angélica Ramos (anexo 2).

\section{Conclusiones}

En conclusión, se podría afirmar que la tutoría entre iguales es una estrategia de aprendizaje eficaz en la formación de los estudiantes normalistas por las múltiples ventajas que ofrece y que se enlistan a continuación: 
1. Se garantiza un acompañamiento a los estudiantes de semestres inferiores con las bondades del aprendizaje entre iguales, es decir, el lenguaje entre los estudiantes así como experiencias similares les permiten mejorar el proceso de planeación, ya que los alumnos tutores reconocen fácilmente las dificultades que enfrentan con la implementación de modalidades de intervención y estrategias básicas para el trabajo sistemático con los niños que se abordan en el quinto y sexto semestres. Del mismo modo se favorece la reflexión-acción y mente abierta, actitudes indispensables en todo docente.

2. Los tutores renuevan su motivación intrínseca al reconocer que requieren fundamentar las observaciones que realizan a sus compañeros lo que permite que se gesten hábitos de autogestión del conocimiento.

3. El aprendizaje cooperativo ofrece a los formadores de docentes la posibilidad no sólo de renovar nuestro actuar en el aula sino de impactar las prácticas docentes de los estudiantes ofreciendo una metodología o técnicas que nos permiten especificar los objetivos académicos, pensar en la mejor manera para organizar a los equipos de trabajo a partir de distinguir sus necesidades educativas y organizar el aula para que las relaciones interpersonales establecidas entre los estudiantes resulten más estables, profundas y comprometidas. Del mismo modo, las relaciones entre el profesorado y los estudiantes son más cercanas y enriquecedoras para ambos.

4. Las metodologías activas implican un proceso de autoevaluación básico en la mejora del actuar docente.

Retomando las experiencias rescatadas en las escuelas de práctica se ha constatado:

- Que las actividades fundamentadas en este tipo de aprendizaje ofrecen al alumno en formación una herramienta para mejorar el control de grupo, pasar del discurso a la práctica de un aprendizaje estratégico que valora la interacción social como un elemento que propicia aprendizajes para la vida, de igual forma se fortalecen las competencias sociales y lingüísticas para la vida en sociedad.

- Los recursos y materiales didácticos pueden potencializarse, permitiendo contar con material más variado. 
- Los niños forman parte activa de su conocimiento y progresan significativamente en la autorregulación de la conducta, igualmente la participación de los padres de familia es sistemática.

Los obstáculos a vencer se distinguieron a partir de la falta de espacios y momentos para desarrollar la tutoría entre iguales por los periodos extensos de tiempo de las estudiantes de séptimo semestre. Tanto en los niños de prescolar y primaria, así como en los estudiantes normalistas se requiere de un periodo de adaptación a la metodología cooperativa ya que durante las primeras sesiones y si las experiencias de aprendizaje anteriores eran inclinadas al tradicionalismo, los estudiantes pueden presentar una baja responsabilidad individual, reducidas participaciones y dificultades con la toma de decisiones.

\section{Bibliografía}

ARONSON, E. (1978). The Jigsaw Classroom. Beberly Hills, California: Sage Publications.

CURTO, C, Gelabert, González, Morales, (2009). Experiencias con Éxito de Aprendizaje Cooperativo en Educación Física. España: INDE.

CUSEO, J. B. (1996). Cooperative learning: A pedagogy for Addressing Contemporary Challanges \& Critical Issues in Higher Education. Marymount College, New Forums Press.

IMBERNÓN, F. (1994): La formación y el desarrollo profesional del profesorado. Barcelona: Grao.

JOHNSON, D. W., \& JOHNSON, R. T. (1989). Cooperative Learning: What Special Education Teachers Needs to Know. Pointer, 33 (2), 5-10. 
LUIS, J.C. (1999): Formación del profesorado y propuestas para mejorar la actuación en Educación Física. Comunicación presentada al XVII Congreso Nacional de Educación Física. Huelva, 1399- 1413.

MAYOR, C. M. (2003). Enseñanza y aprendizaje en la Educación Superior. Barcelona: Octaedro-EUB.

MONEREO, C., \& POZO, J. I. (2003): La universidad ante la nueva cultura educativa. Madrid: Ed. Síntesis.

OVEJERO, A. (1990). El aprendizaje cooperativo. Una alternativa eficaz a la enseñanza tradicional. Barcelona: P.P.U.

VELÁZQUEZ, C. (2010). Aprendizaje Cooperativo en Educación Física. España: INDE.

ZABALZA, M. A. (2000). La enseñanza universitaria: el escenario y los protagonistas. Madrid: Narcea.

Anexos (tablas y gráficos)

PROMEDIOS DE ACIERTOS EN EL EXAMEN EN EL ESTADO DE

PUEBLA

LICENCIATURA EN EDUCACIÓN SECUNDARIA 2010 FÍSICA

SEXTO SEMESTRE

\begin{tabular}{|c|c|c|c|c|c|c|c|c|c|}
\hline \multirow[b]{2}{*}{ Sustentantes } & \multirow[b]{2}{*}{$\begin{array}{l}\text { Promedio } \\
\text { Global }\end{array}$} & \multicolumn{5}{|c|}{ Formación común } & \multicolumn{3}{|c|}{ Formadión especifica } \\
\hline & & Total & $\begin{array}{l}\text { Habiidades } \\
\text { intelectuales }\end{array}$ & $\begin{array}{l}\text { Propósitos y contenidos } \\
\text { de la educacón } \\
\text { secundaria }\end{array}$ & $\begin{array}{l}\text { Competencias } \\
\text { didácticas } \\
\text { generales }\end{array}$ & \begin{tabular}{|l|} 
Identidad \\
profesional
\end{tabular} & Total & \begin{tabular}{|l|} 
Contenidos \\
diciplinarios
\end{tabular} & $\begin{array}{l}\text { Competencias } \\
\text { didádicas } \\
\text { especificas }\end{array}$ \\
\hline 13 & 47.80 & 47.33 & 47.20 & 45.75 & 49.70 & 46.89 & 48.25 & 45.47 & 51.14 \\
\hline
\end{tabular}

Tabla 1. Resultados del Examen de conocimientos aplicado por CENEVAL 
PROMEDIOS DE ACIERTOS EN EL EXAMEN EN EL ESTADO DE

PUEBLA

LICENCIATURA EN EDUCACIÓN PREESCOLAR 2010*

SEXTO SEMESTRE

\begin{tabular}{|c|c|c|c|c|c|}
\hline Sustentantes & $\begin{array}{l}\text { Promedio } \\
\text { Global }\end{array}$ & $\begin{array}{l}\text { Propósitos y contenidos } \\
\text { de la educación Preescolar }\end{array}$ & $\begin{array}{l}\text { Competencias } \\
\text { didácticas }\end{array}$ & $\begin{array}{l}\text { Habilidades } \\
\text { intelectuales }\end{array}$ & $\begin{array}{l}\text { Percepción y respuesta al } \\
\text { entorno de la escuela }\end{array}$ \\
\hline & 54.11 & 51.07 & 56.20 & 53.77 & 56.14 \\
\hline
\end{tabular}

\begin{tabular}{|l|l||l||}
\hline & $\begin{array}{l}\text { "Logro obtenidos } \\
\text { durante la sesión" }\end{array}$ & "Dificultades durante la sesión" \\
\hline
\end{tabular}

PROMEDIOS DE ACIERTOS EN EL EXAMEN EN EL ESTADO DE

PUEBLA

LICENCIATURA EN EDUCACIÓN SECUNDARIA 2010 FÍSICA

OCTAVO SEMESTRE

\begin{tabular}{|c|c|c|c|c|c|c|c|c|c|}
\hline \multirow[b]{2}{*}{ Sustentantes } & \multirow[b]{2}{*}{$\begin{array}{l}\text { Promedio } \\
\text { Global }\end{array}$} & \multicolumn{5}{|c|}{ Formación común } & \multicolumn{3}{|c|}{ Formación específica } \\
\hline & & Total & $\begin{array}{l}\text { Habiidades } \\
\text { intelectuales }\end{array}$ & $\begin{array}{l}\text { Propósitos y contenidos } \\
\text { de la educacoón } \\
\text { secundaria } \\
\end{array}$ & $\begin{array}{l}\text { Competendias } \\
\text { didácticas } \\
\text { generales }\end{array}$ & \begin{tabular}{|l|} 
Identidad \\
profesional
\end{tabular} & Total & $\begin{array}{l}\text { Contenidos } \\
\text { diciplinarios }\end{array}$ & $\begin{array}{l}\text { Competencias } \\
\text { didácticas } \\
\text { especificas }\end{array}$ \\
\hline 22 & 43.45 & 42.77 & 38.22 & 40.91 & 44.58 & 42.43 & 44.09 & 41.40 & 46.89 \\
\hline
\end{tabular}

PROMEDIOS DE ACIERTOS EN EL EXAMEN EN EL ESTADO DE

PUEBLA

LICENCIATURA EN EDUCACIÓN PREESCOLAR 2010

OCTAVO SEMESTRE

\begin{tabular}{|c|c|c|c|c|c|}
\hline Sustentantes & $\begin{array}{l}\text { Promedio } \\
\text { Global }\end{array}$ & $\begin{array}{l}\text { Propósitos y contenidos } \\
\text { de la educación Preescolar }\end{array}$ & $\begin{array}{l}\text { Competencias } \\
\text { didácticas }\end{array}$ & $\begin{array}{l}\text { Habilidades } \\
\text { intelectuales }\end{array}$ & $\begin{array}{l}\text { Percepción y respuesta al } \\
\text { entorno de la escuela }\end{array}$ \\
\hline & 57.20 & 53.84 & 59.05 & 56.96 & 59.52 \\
\hline
\end{tabular}

Anexo 2. Diario de la estudiante Julieta Carmona, ciclo escolar 2010-2011 de la licenciatura en educación preescolar, realizado en el segundo periodo de práctica docente. 


\begin{tabular}{|c|c|c|}
\hline Análisis & Soy mejor en.. & $\begin{array}{l}\text { Mis debilidades son... } \\
\text { Mi tono de voz }\end{array}$ \\
\hline Análisis & $\begin{array}{l}\text { "Apoyos" } \\
\text { El material didáctico }\end{array}$ & $\begin{array}{l}\text { "Peligros" } \\
\text { No preveer los materiales para } \\
\text { trabajar }\end{array}$ \\
\hline Fecha & \multicolumn{2}{|l|}{11 de Octubre de 2010} \\
\hline $\begin{array}{l}\text { Los niños empe } \\
\text { y se me quedab } \\
\text { maestra Sofia } \\
\text { saludaban cuand } \\
\text { sus cosas afuera } \\
\text { tomaran asient } \\
\text { instrucciones, no } \\
\text { para ir a ceremon } \\
\text { La ceremonia de } \\
\text { maestra Chuy, } \\
\text { descubrimiento } \\
\text { una representaci } \\
\text { los demás niño } \\
\text { ceremonia mis c } \\
\text { maestra para } \\
\text { ceremonia y real } \\
\text { Cuando termin } \\
\text { niños que nos d } \\
\text { A, pera ver unos } \\
\text { Salude a mis a } \\
\text { actividad con } 1 \\
\text { inconveniente } \\
\text { reconocer mi me } \\
\text { detectaba los cab }\end{array}$ & 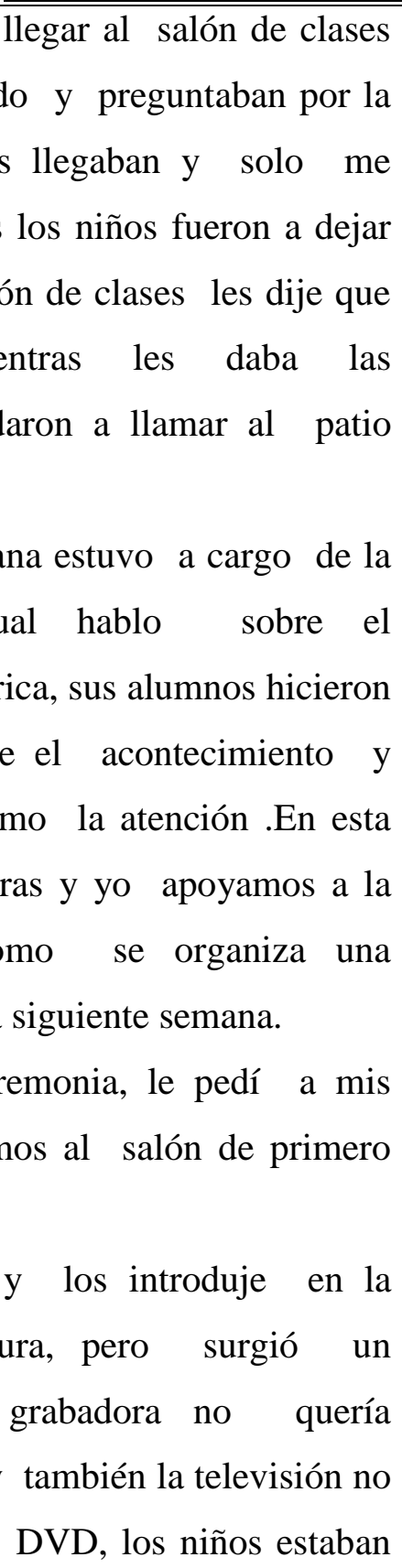 & $\begin{array}{l}\text { Preguntas / Comentarios } \\
\text { En este día me sentí muy } \\
\text { nerviosa,siento que influyo cuando } \\
\text { la grabadora y el televisor no } \\
\text { querían funcionar. Aunque la verdad } \\
\text { siento que si la regué en lo preparar } \\
\text { el material con anticipación, como } \\
\text { es ver si funcionan los aparatos del } \\
\text { jardín de niños. } \\
\text { Algo que me gusto mucho de este } \\
\text { dia es que Ruben me trabajo muy } \\
\text { bien, fue uno de los niños que más } \\
\text { me participo, al principio era de lo } \\
\text { que mas le temía debido que en días } \\
\text { anteriores estaba muy inquieto. }\end{array}$ \\
\hline
\end{tabular}




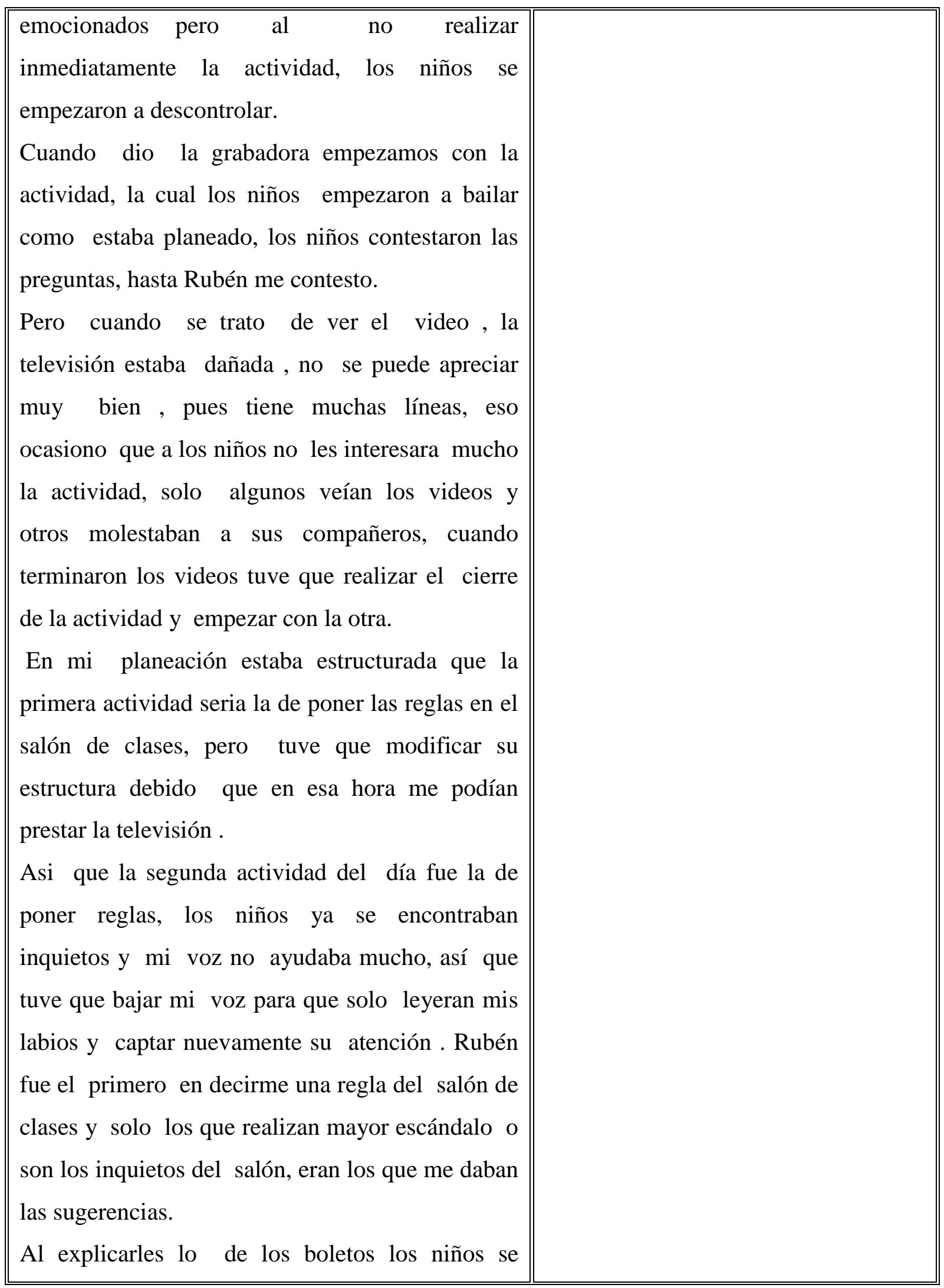




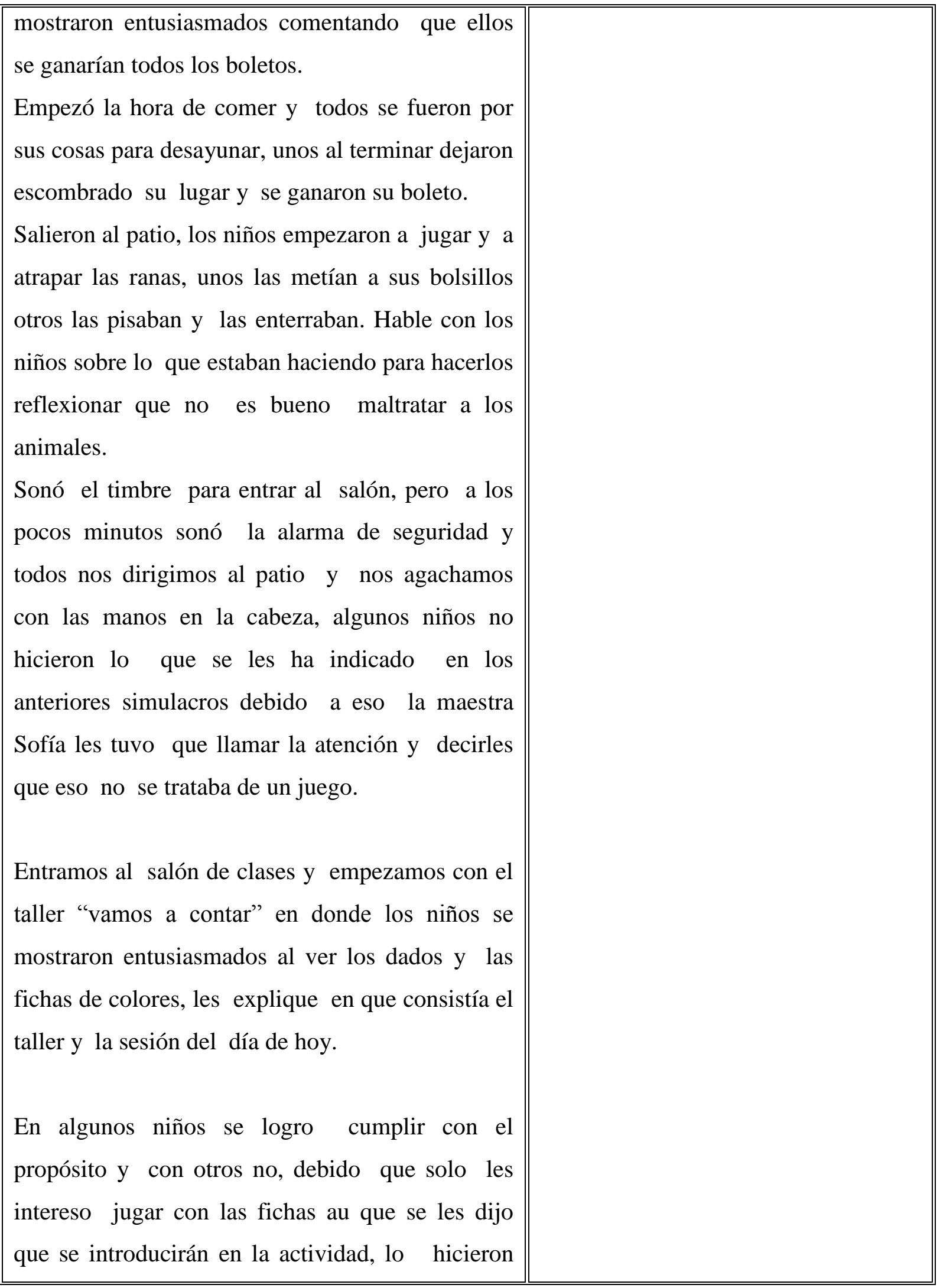




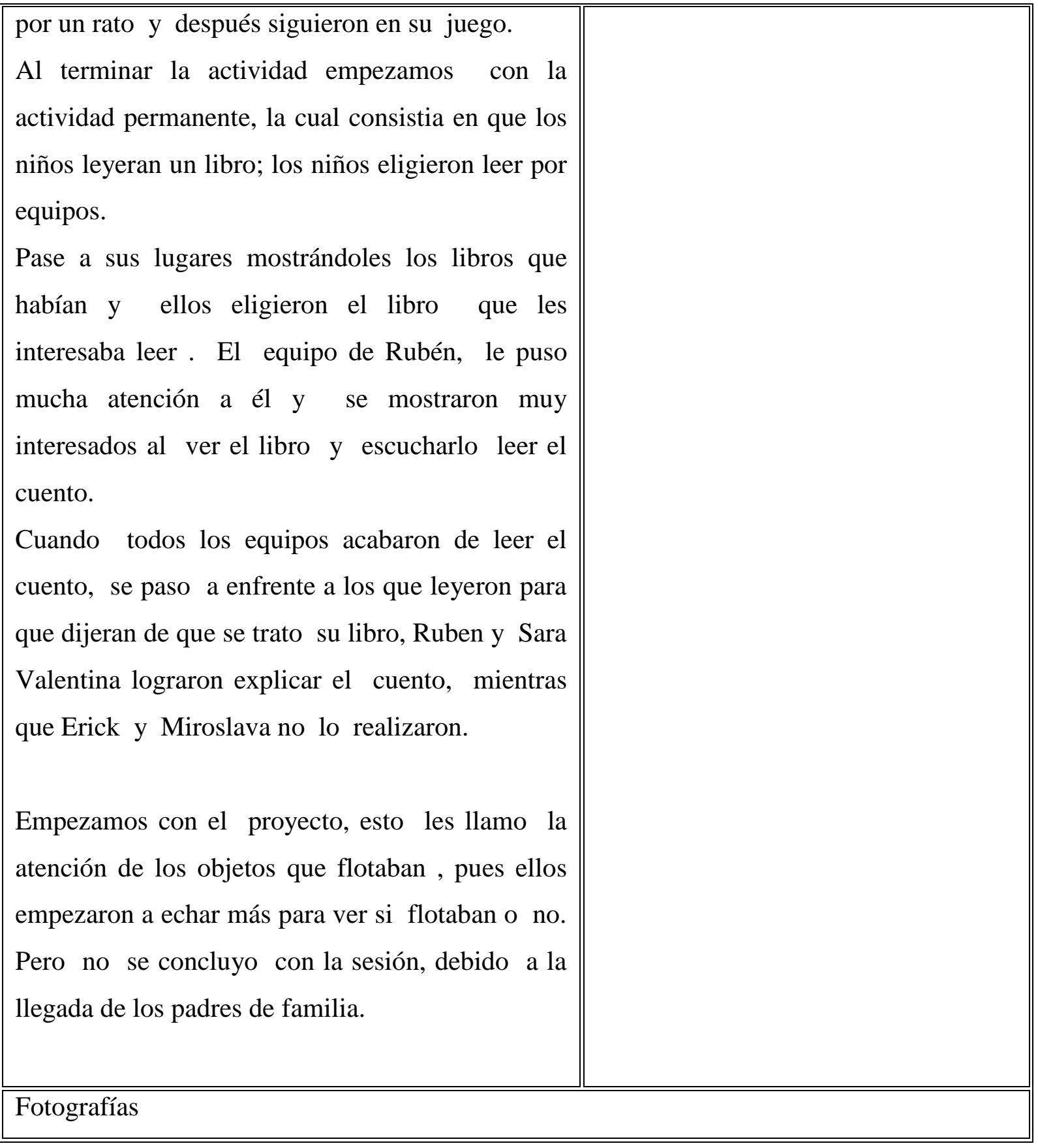




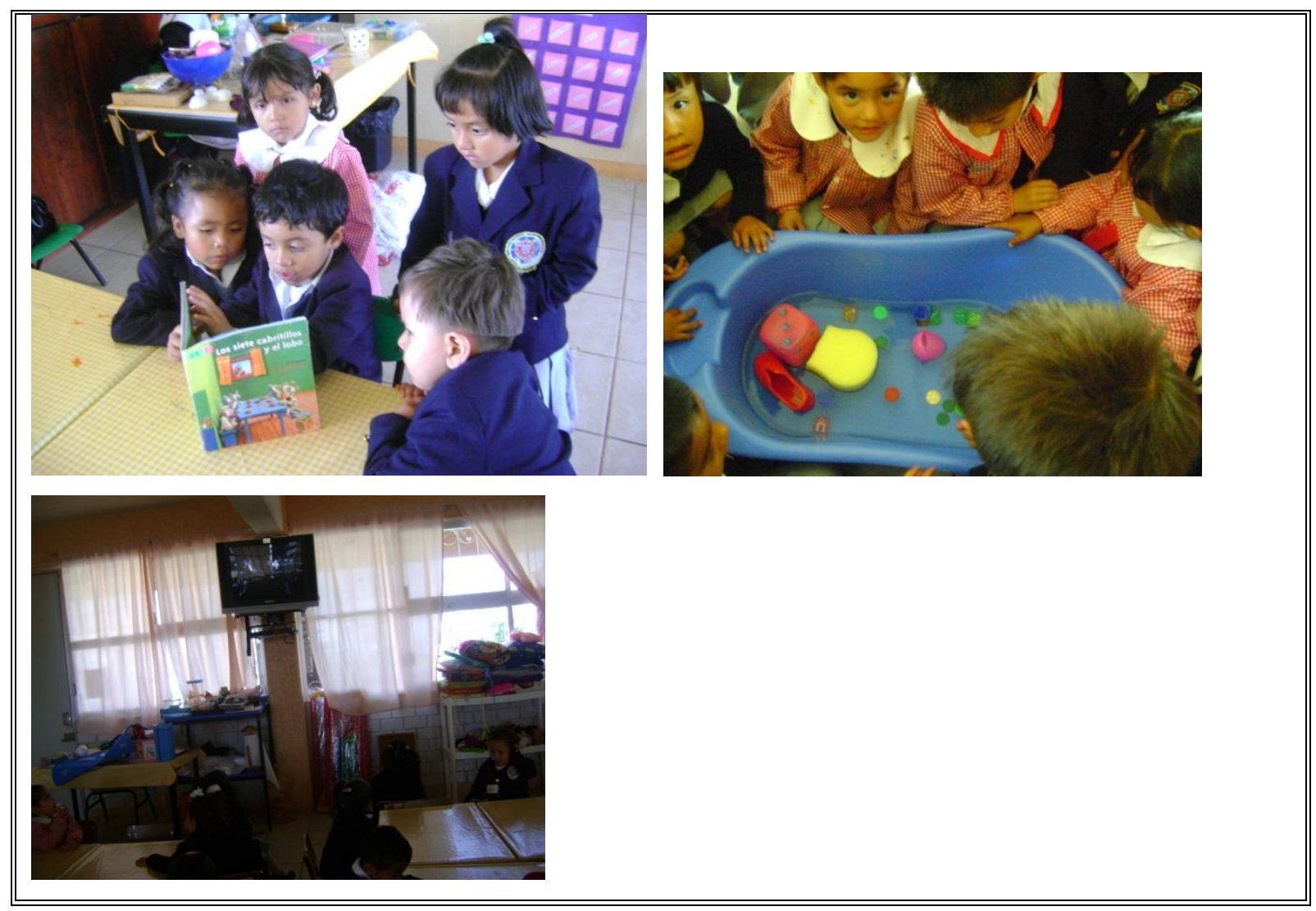


Anexo 2. Planeación de la estudiante Angélica Ramos, basada en el puzzle

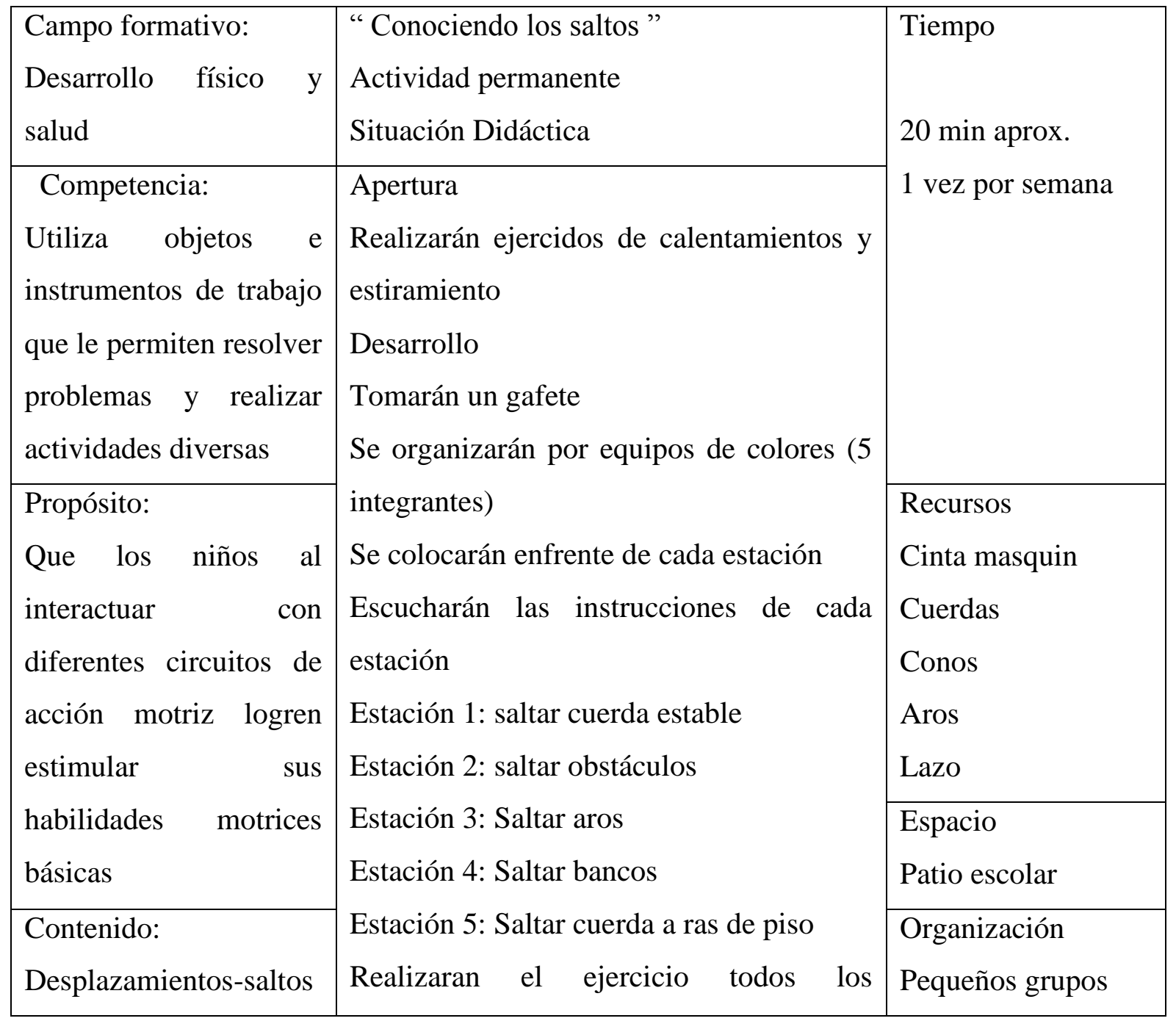




\begin{tabular}{|c|c|c|}
\hline $\begin{array}{l}\text { Estrategia Básica: } \\
\text { El aprendizaje a través } \\
\text { del juego }\end{array}$ & $\begin{array}{l}\text { integrantes del equipo pasando dos veces } \\
\text { por la estación } \\
\text { Formarán equipos multicolor } \\
\text { Pasarán por cada circuito donde el } \\
\text { especialista les explicara cómo deben } \\
\text { pasar por la estación } \\
\text { Cierre } \\
\text { Realizarán ejercicios de relajación } \\
\text { Comentarán ¿qué es lo que hicieron en } \\
\text { cada estación? ¿con que materiales } \\
\text { trabajaron? ¿fue difícil explicarle a sus } \\
\text { compañeros como pasar en cada } \\
\text { estación? } \\
\text { Mencionarán que partes del cuerpo } \\
\text { utilizaron para realizar cada ejercicio }\end{array}$ & $\begin{array}{l}\text { Evaluación: } \\
\text { Los niños: } \\
\text {-los niños } \\
\text { respetaban el turno } \\
\text { que les correspondía } \\
\text {-se apoyaban para } \\
\text { realizar } \\
\text { ejercicios } \\
\text {-cuando los } \\
\text { mesclaron } \\
\text { reaccionaron } \\
\text { niños los } \\
\text { que } \\
\text { instrucciones } \\
\text { compañeros }\end{array}$ \\
\hline
\end{tabular}

